\title{
Research on Shifting Control Method of Positive Independent Mechanical Split Path Transmission for the Starting Gear
}

\author{
JunQiang Xi, ${ }^{1}$ JianMin Meng, ${ }^{2}$ and HuiYan Chen ${ }^{1}$ \\ ${ }^{1}$ Science and Technology on Vehicle Transmission Laboratory, Beijing Institute of Technology, Beijing 100081, China \\ ${ }^{2}$ Patent Examination Cooperation Center of the Patent Office, SIPO, Beijing 100081, China \\ Correspondence should be addressed to JunQiang Xi; xijunqiang@bit.edu.cn
}

Received 24 October 2013; Revised 21 November 2013; Accepted 21 November 2013

Academic Editor: Hui Zhang

Copyright (C) 2013 JunQiang Xi et al. This is an open access article distributed under the Creative Commons Attribution License, which permits unrestricted use, distribution, and reproduction in any medium, provided the original work is properly cited.

To realize a smooth and quick shift of the positive independent mechanical split path transmission (PIMSPT) equipped with automatic shifting control system (ASCS), the research on the feasibility of improving shift quality by dynamic and cooperative controlling engine, steering clutches, and brakes has been conducted. The shifting control method suited to starting gear of PIMSPT has been proposed. The control method is based on control parameters, such as the driving shaft speed and its derivative. The control laws of steering clutches and brakes are presented during each gear and stage of shifting. Bench and road test results show that the proposed shifting control method can not only shorten the shift time, but also decrease the jerk of shifting effectively.

\section{Introduction}

During the straight driving process of a vehicle equipped with PIMSPT, power from the engine is transmitted through the shifting mechanism and steering mechanism. The splitting power is converged by planet gear trains on each side of the transmission and then outputs to driving wheels. Thus, the shifting mechanism only transmits part of the engine torque, which means that the torque transmitted by the shifting mechanism can be decreased. As a result, the power density of the transmission can be improved. So, the PIMSPT is widely used in many kinds of track vehicles in the world [1-3].

Figure 1 shows a schematic diagram of a PIMSPT and its power transmission path. We can see that the PIMSPT shares the same shifting components with the fixed shaft transmission, although its structure is very different from the latter. Because of the difference of the structures, both the driving shaft and the countershaft of the PIMSPT are rotating when the vehicle stops, which leads to longer shifting time of starting gear and causes more shifting noise. This paper takes the PIMSPT shown in Figure 1 as a research subject and conducts research on the shifting control method for starting gear.

\section{Kinematics and Dynamics Analysis}

2.1. Dynamic Model of Neutral Position. Assuming that the gear shift of transmission is in the neutral position before launching. First, we should separate the main clutch completely. The planetary carriers are static now. The driving shaft and the countershaft of the transmission are decelerated by the resistant torque produced by the oil. Considering the structure of the PIMSPT, we can set up the kinematic model and the dynamic model of the transmission in the condition that the steering clutches or steering brakes are engaged, according to their status on both sides.

2.1.1. Analysis of Steering Clutches Engagement. When the clutches on both sides are engaged, respectively, the counter shaft, steering clutches, and sun gear shaft can be regarded as a whole. And we can assume that the rotational inertia of counter shaft can be added to the sun gear shaft. The motion state of each transmission component can be seen in Figures 2 and 3 .

In Figures 2 and 3, $T_{L}, T_{R}$ represent the resistance imposed on each side of the planet carrier, respectively, and are caused 


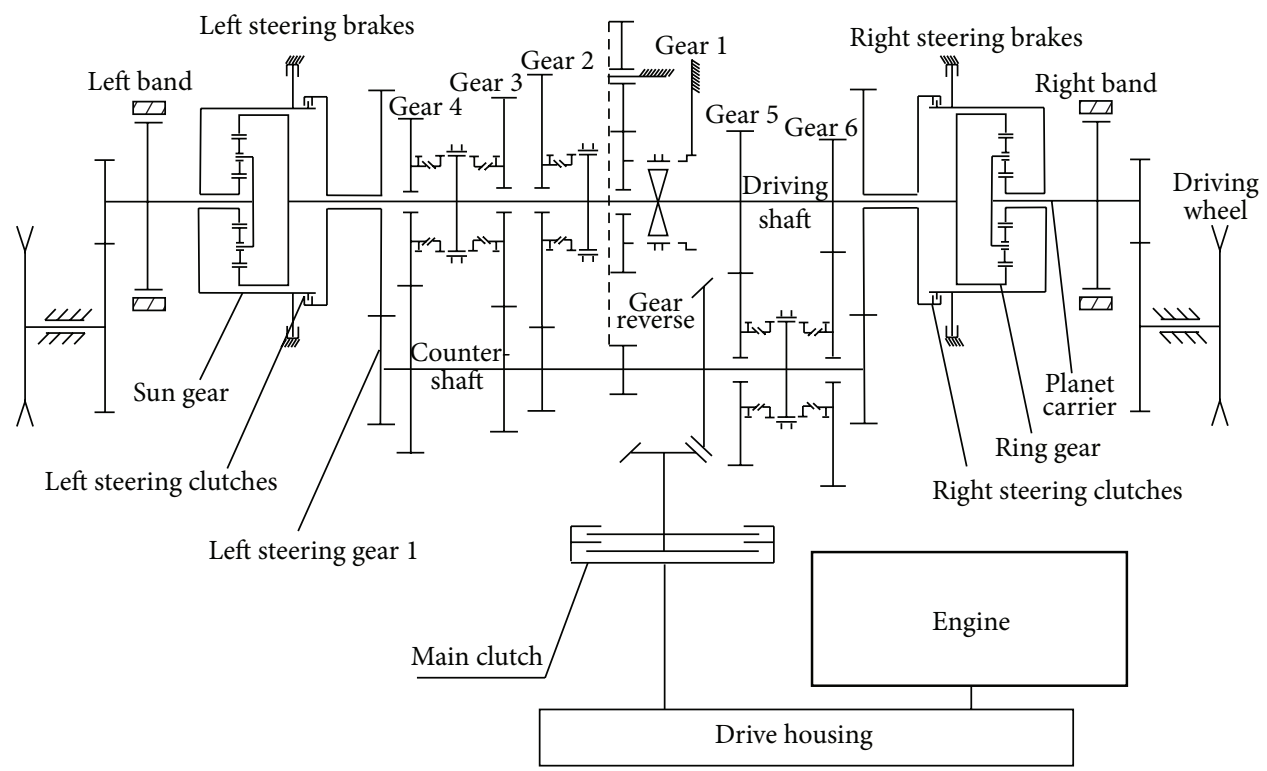

FIgURE 1: The structure of the PIMSPT and the transmit path of the power.

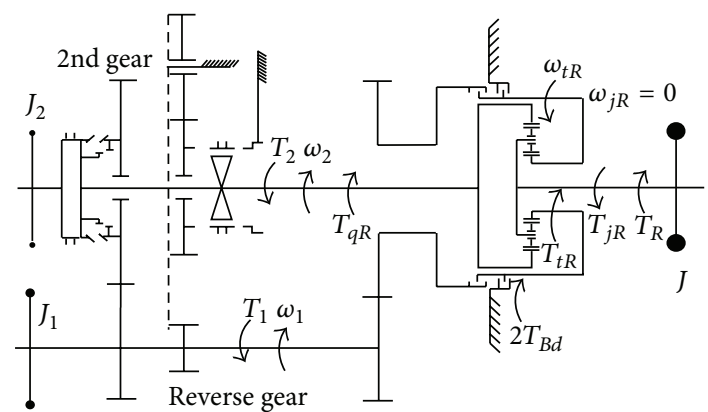

FIGURE 2: The diagram of dynamics analysis of the components of the gear box in the condition of the steering clutches engaged.

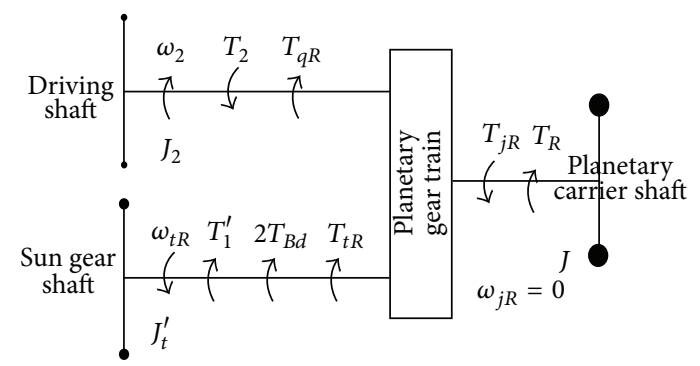

FIgURE 3: The diagram of equivalent kinematics and dynamics analysis of the components of the gear box in the condition of the steering clutches engaged.

by road resistance, $\mathrm{N} \cdot \mathrm{m} ; \omega_{1}, \omega_{2}$ represent the angular speed of the counter shaft and the driving shaft, respectively, $\mathrm{rad} / \mathrm{s}$; $\omega_{t L} \omega_{t R}$ represent the angular speed of the sun gears, $\mathrm{rad} / \mathrm{s}$; $\omega_{z L}, \omega_{z R}$ represent the angular speed of the steering clutches, $\mathrm{rad} / \mathrm{s} ; J_{1}, J_{2}$ represent the sum of the rotational inertia of the counter shaft and the driving shaft with some rotating components and the equivalent rotational inertia of the free gears on their own shaft, respectively, $\mathrm{kg} \cdot \mathrm{m}^{2} ; J$ represents the rotational inertia converted to the planet carrier shaft due to the mass of vehicle, $\mathrm{kg} \cdot \mathrm{m}^{2}$. $J_{t}^{\prime}$ represents the rotational inertia converted to the sun gear shaft by the counter shaft, $J_{t}^{\prime}=i_{z}^{2} J_{1}+2 J_{t}+2 J_{c}, \mathrm{~kg} \cdot \mathrm{m}^{2}$, where $J_{t}$ and $J_{c}$ represent the rotational inertia of the sun gears and the steering clutch, respectively, $\mathrm{kg} \cdot \mathrm{m}^{2}$. Because $J_{1}, J_{2}$ are much greater than $J_{t}$, $J_{c}$, we can assume that $J_{t}^{\prime}=i_{z}^{2} J_{1} ; T_{1}$ and $T_{2}$ represent the resistance moment of the counter shaft and the driving shaft, respectively, $\mathrm{N} \cdot \mathrm{m} ; T_{t R}, T_{q R}$, and $T_{j R}$ represent, respectively the resistance moment on the sun gear shaft, driving shaft, and the planet carrier forced by the planet gears, $\mathrm{N} \cdot \mathrm{m} ; T_{B d}$ represents the resistance moment on the sun gear shaft forced by the steering brakes of a single side, $\mathrm{N} \cdot \mathrm{m} ; T_{1}^{\prime}$ represents the resistance moment on the sun gear shaft by $T_{1}, T_{1}^{\prime}=i_{z} T_{1}$, $\mathrm{N} \cdot \mathrm{m} ; T_{d}$ represents the towing torque when the multiplate wet clutch is separating, $\mathrm{N} \cdot \mathrm{m}$.

Establishing the equivalent kinetics equations of the sun gear shaft and the driving shaft according to the force analysis of Figure 3:

$$
\begin{gathered}
J_{t}^{\prime} \dot{\omega}_{t R}=T_{t R}+T_{1}^{\prime}+2 T_{B d}, \\
J_{2} \dot{\omega}_{2}=T_{q R}-T_{2} .
\end{gathered}
$$

In (1), $\dot{\omega}_{t R}, \dot{\omega}_{2}$ represent the angular acceleration of the sun gear shaft and the driving shaft, respectively, $\mathrm{rad} / \mathrm{s}^{2}$. And $\dot{\omega}_{R}=$ $\dot{\omega}_{1} / i_{z}, \dot{\omega}_{1}$ represents the angular acceleration of the counter shaft, $\mathrm{rad} / \mathrm{s}^{2}$.

The speed and resistance moment of a single planetary gear set satisfy the following equations:

$$
\begin{gathered}
\omega_{t}+k \omega_{q}=(1+k) \omega_{j}, \\
\dot{\omega}_{t}+k \dot{\omega}_{q}=(1+k) \dot{\omega}_{j}, \\
T_{t}: T_{q}: T_{j}=1: k:(1+k) .
\end{gathered}
$$




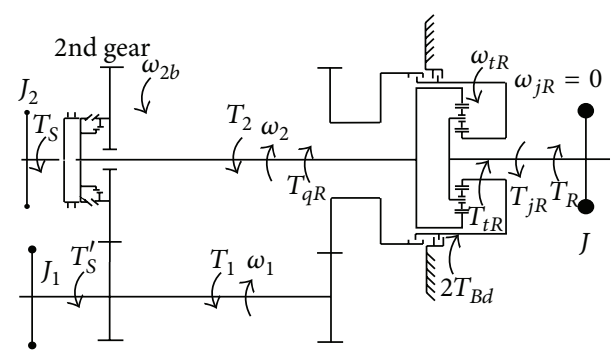

FIGURE 4: The diagram of kinematics and dynamics analysis of the components of the gear box before launching in the condition of the steering brakes engaged.

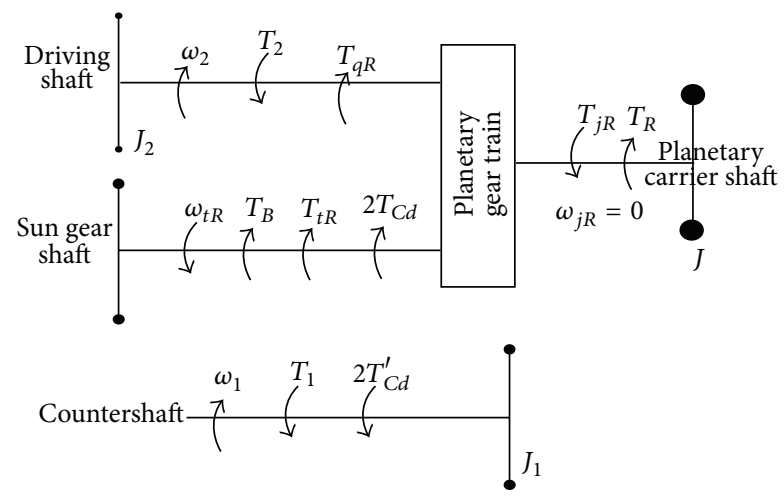

FIgURE 5: The diagram of the equivalent kinematics and the dynamics analysis of the components of the gear box before launching in the condition of the steering brakes engaged.

In (2), $\omega_{t}, \omega_{q}$, and $\omega_{j}$ represent the angular speed of the sun gear shaft, ring shaft, and the planet carrier, respectively, $\mathrm{rad} / \mathrm{s} ; \dot{\omega}_{t}, \dot{\omega}_{q}, \dot{\omega}_{j}$ represent the angular acceleration of them, $\mathrm{rad} / \mathrm{s}^{2}$; and $T_{t}, T_{q}$, and $T_{j}$ represent the resistance moment on them, $\mathrm{N} \cdot \mathrm{m}$.

When $\omega_{j}=0, \dot{\omega}_{j}=0$, we can calculate the angular acceleration of the counter shaft and the driving shaft according to (1) and (2):

$$
\begin{gathered}
{\left[\begin{array}{c}
\dot{\omega}_{1} \\
\dot{\omega}_{2}
\end{array}\right]=\frac{-1}{J_{2}+k^{2} i_{z}^{2} J_{1}}\left[\begin{array}{ccc}
\left(k i_{z}\right)^{2} & k i_{z} & 2 k^{2} i_{z} \\
k i_{z} & 1 & 2 k
\end{array}\right]\left[\begin{array}{c}
T_{1} \\
T_{2} \\
T_{B d}
\end{array}\right],} \\
T_{j R}=\frac{1+k}{J_{2}+k^{2} i_{z}^{2} J_{1}}\left[\begin{array}{lll}
-i_{z} J_{2} & k i_{z}^{2} J_{1} & -2 J_{2}
\end{array}\right]\left[\begin{array}{c}
T_{1} \\
T_{2} \\
T_{B d}
\end{array}\right] .
\end{gathered}
$$

2.1.2. Analysis of Steering Brakes Combination. When the steering brakes of both sides are engaged and the steering clutches of both sides are released, the counter shaft and the sun gears move independently. The motion state and stress state of each component are shown in Figures 4 and 5.

In Figures 4 and $5, T_{C d}$ represents the towing torque of the steering clutches, $\mathrm{N} \cdot \mathrm{m} ; \mathrm{T}_{C d}^{\prime}$ represents the equivalent torque on the counter shaft by $T_{C d}, T_{C d}^{\prime}=T_{C d} / i_{z}, \mathrm{~N} \cdot \mathrm{m} . T_{B}$ represents the braking torque on the sun gear shaft forced by the steering brakes, $\mathrm{N} \cdot \mathrm{m}$.

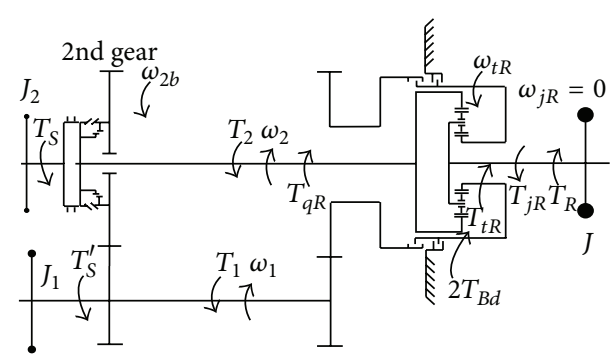

FIGURE 6: The diagram of the kinematics and dynamics analysis of the components of the gear box in the synchronizing stage of 2nd gear in the condition of the steering brakes being engaged.

Establishing the kinetics equations of the counter shaft, the driving shaft, and the sun gear shaft according to the force analysis of Figure 5,

$$
\begin{gathered}
J_{1} \dot{\omega}_{1}=-T_{1}-2 T_{C d}^{\prime}, \\
J_{t} \dot{\omega}_{t R}=T_{t R}+T_{B}+2 T_{C d}, \\
J_{2} \dot{\omega}_{2}=T_{q R}-T_{2} .
\end{gathered}
$$

When $\omega_{j}=0, \dot{\omega}_{j}=0$, we can calculate the following results according to (2) and (5):

$$
\begin{aligned}
{\left[\begin{array}{l}
\dot{\omega}_{1} \\
\dot{\omega}_{2}
\end{array}\right]=} & -\left[\begin{array}{cccc}
\frac{1}{J_{1}} & 0 & 0 & \frac{2}{i_{z} J_{1}} \\
0 & \frac{1}{\left(J_{2}+k^{2} J_{t}\right)} & \frac{k}{\left(J_{2}+k^{2} J_{t}\right)} & \frac{2 k}{\left(J_{2}+k^{2} J_{t}\right)}
\end{array}\right] \\
& \times\left[\begin{array}{c}
T_{1} \\
T_{2} \\
T_{B} \\
T_{C d}
\end{array}\right], \\
T_{j R} & =\frac{1+k}{J_{2}+k^{2} J_{t}}\left[\begin{array}{lll}
k J_{t} & -J_{2} & -2 J_{2}
\end{array}\right]\left[\begin{array}{c}
T_{2} \\
T_{B} \\
T_{C d}
\end{array}\right] .
\end{aligned}
$$

2.1.3. Analysis Conclusion. By comparing (3) with (6), we know that the angular speeds of the counter shaft and the driving shaft decrease faster when the main clutch is released and the steering brakes are engaged.

2.2. Dynamic Model of Shifting Process. Taking the shifting process of 2nd gear as an example, synchronizer is used during this shifting process. According to Figure 6, we know that when the vehicle is launching with 2 nd gear, the synchronizer rotates in opposite direction to the 2 nd gear and the angular speeds are $\omega_{2}$ and $\omega_{2 b}=\omega_{1} / i_{2}$, respectively. When they reach the same speed, the angular speed difference value of the synchronizer and the 2 nd gear should be zero.

We divide the shifting process into two stages: synchronizer gap elimination and synchronizer working. And then we analyze the conditions of the steering clutches and the steering brakes of both sides when they are engaged. We have analyzed the former condition in Section 2.1. 


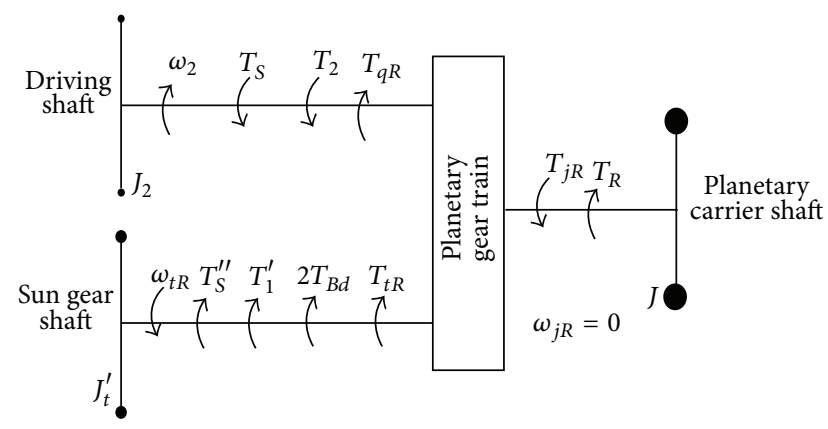

FIGURE 7: The diagram of the equivalent kinematics and dynamics analysis of the components of the gear box in the synchronizing stage of 2 nd gear in the condition of steering brakes being engaged.

\subsubsection{Analysis of Shifting Process in the Condition of Steering} Clutches Engaged. Under the action of shifting force, there are equal and opposite torque on the friction cone of the synchronizer and the driving gear. The synchronizing torque on the driven gear in 2nd gear is transmitted to the counter shaft by the constant mesh gear of 2 nd gear. The motion state and stress state of each component in this condition can be seen in Figure 6.

In Figures 6 and 7, $T_{s}$ represents the torque on the synchronizer, $\mathrm{N} \cdot \mathrm{m} ; T_{s}^{\prime}$ represents the torque transmitted to the counter shaft, $T_{s}^{\prime}=T_{s} / i_{2}, \mathrm{~N} \cdot \mathrm{m} ; T_{s}^{\prime \prime}$ represents the equivalent torque on the sun gear shaft by $T_{s}, T_{s}^{\prime \prime}=T_{s} i_{z} / i_{2}$, $\mathrm{N} \cdot \mathrm{m}$. Other parameters have the same meaning as mentioned previously.

Establishing the kinetics equations of the sun gear shaft, driving shaft, and the sun gear shaft according to the force analysis of Figure 7,

$$
\begin{gathered}
J_{t}^{\prime} \dot{\omega}_{t R}=T_{t R}+T_{1}^{\prime}+2 T_{B d}+T_{s}^{\prime \prime}, \\
J_{2} \dot{\omega}_{2}=T_{q R}-T_{2}-T_{s} .
\end{gathered}
$$

According to (8) and (9), we can calculate the angular accelerations $\left(\dot{\omega}_{1+2}, \dot{\omega}_{2 t 2}\right)$ of the counter shaft and the driving shaft during synchronization:

$$
\begin{aligned}
{\left[\begin{array}{c}
\dot{\omega}_{1+2} \\
\dot{\omega}_{2 t 2}
\end{array}\right]=} & \frac{-1}{J_{2}+k^{2} i_{z}^{2} J_{1}} \\
& \times\left[\begin{array}{cccc}
\left(k i_{z}\right)^{2} & k i_{z} & k^{2} i_{z} & k i_{z}+\frac{\left(k i_{z}\right)^{2}}{i_{2}} \\
k i_{z} & 1 & k & 1+\frac{k i_{z}}{i_{2}}
\end{array}\right]\left[\begin{array}{c}
T_{1} \\
T_{2} \\
2 T_{B d} \\
T_{s}
\end{array}\right] .
\end{aligned}
$$

When the angular acceleration is constant, the relation of the angular speed and the angular acceleration is as follows:

$$
\dot{\omega}=\frac{\left(\omega_{e}-\omega_{b}\right)}{t} .
$$

In (11), $t$ represents the action time, $s ; \omega_{b}$ represents the angular speed when the angular acceleration has a certain value, $\mathrm{rad} / \mathrm{s}$; and $\omega_{e}$ represents the angular speed when the angular acceleration stays zero, $\mathrm{rad} / \mathrm{s}$.

If $\omega_{1 t 0}, \omega_{2 t 0}$ represent the angular speeds of the counter shaft and the driving shaft in $t_{0}$, then we can calculate the angular speeds $\left(\omega_{1 t 1}, \omega_{2 t 1}\right)$ of them when the synchronization process begins.

According to (9), (10), and (11), the time of the stage before the synchronization process can be calculated, $t_{01}=t_{1}-t_{0}$, $t_{12}=t_{2}-t_{1}$. And we can also get the functional relationship of the synchronizing torque, the angular speeds of the counter shaft, and the driving shaft when synchronization begins and the synchronization time is

$$
\begin{aligned}
& t_{01}=\left\{\begin{array}{l}
\frac{\left(\omega_{1 t 0}-\omega_{1 t 1}\right)\left(J_{2}+k^{2} i_{z}^{2} J_{1}\right)}{\left[\left(k i_{z}\right)^{2} T_{1}+k i_{z} T_{2}+2 k^{2} i_{z} T_{B d}\right]}, \\
\frac{\left(\omega_{2 t 0}-\omega_{2 t 1}\right)\left(J_{2}+k^{2} i_{z}^{2} J_{1}\right)}{\left(k i_{z} T_{1}+T_{2}+2 k T_{B d}\right)},
\end{array}\right. \\
& t_{12}=\left\{\begin{array}{c}
\omega_{1 t 1}\left(k^{2} i_{z}^{2} J_{1}+J_{2}\right) \\
\times\left[\left(k i_{z}\right)^{2} T_{1}+k i_{z} T_{2}+2 k^{2} i_{z} T_{B d}\right. \\
\left.+\left(k i_{z}+\frac{\left(k i_{z}\right)^{2}}{i_{2}}\right) T_{s}\right]^{-1}, \\
\omega_{2 t 1}\left(k^{2} i_{z}^{2} J_{1}+J_{2}\right) \\
\frac{\left[k i_{z} T_{1}+T_{2}+2 k T_{B d}+\left(1+k i_{z} / i_{2}\right) T_{s}\right]}{},
\end{array}\right. \\
& T_{s}=\left\{\begin{array}{l}
\frac{\left(k^{2} i_{z}^{2} J_{1}+J_{2}\right) \omega_{1 t 1}}{\left[k i_{z}+\left(k i_{z}\right)^{2} / i_{2}\right] t_{12}} \\
-\frac{1}{k i_{z}+\left(k i_{z}\right)^{2} / i_{2}}\left[\begin{array}{lll}
\left(k i_{z}\right)^{2} & k i_{z} & 2 k^{2} i_{z}
\end{array}\right]\left[\begin{array}{c}
T_{1} \\
T_{2} \\
T_{B d}
\end{array}\right], \\
\frac{\left(k^{2} i_{z}^{2} J_{1}+J_{2}\right) \omega_{2 t 1}}{\left(1+k i_{z} / i_{2}\right) t_{12}} \\
-\frac{1}{\left(1+k i_{z} / i_{2}\right)}\left[\begin{array}{lll}
k^{2} i_{z} & 1 & 2 k
\end{array}\right]\left[\begin{array}{c}
T_{1} \\
T_{2} \\
T_{B d}
\end{array}\right] .
\end{array}\right.
\end{aligned}
$$

Based on energy conservation law, the friction work produced in the process of synchronization can be calculated by the following:

$$
\begin{aligned}
W_{s}= & \frac{1}{2} J_{1} \omega_{1 t 1}^{2}+\frac{1}{2} J_{2} \omega_{2 t 1}^{2}-\left(T_{1} \omega_{1 t 1}+T_{2} \omega_{2 t 1}\right) \\
& -\int_{t 1}^{t 2} 2 T_{B d} \omega_{1 t 1} d t .
\end{aligned}
$$

In this equation, $W_{s}$ represents the friction work of the synchronizer, $J$.

From (12), we know that the friction work of the synchronizer is directly proportional to the angular speed of the counter shaft and the driving shaft when the synchronization begins. Thus, the friction work can be reduced by reducing $\omega_{1 t 1}$ and $\omega_{2 t 1}$. When the steering clutches of both sides are 


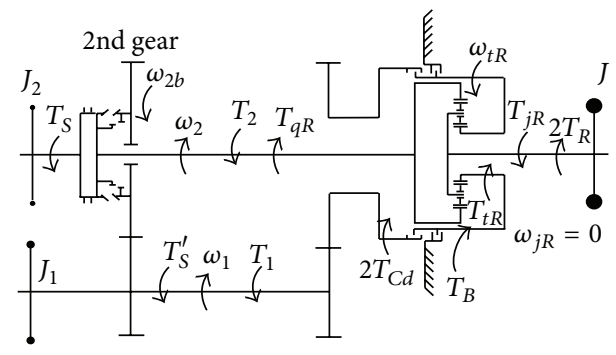

FIGURE 8: The diagram of the kinematics and dynamics analysis of the components of the gear box.

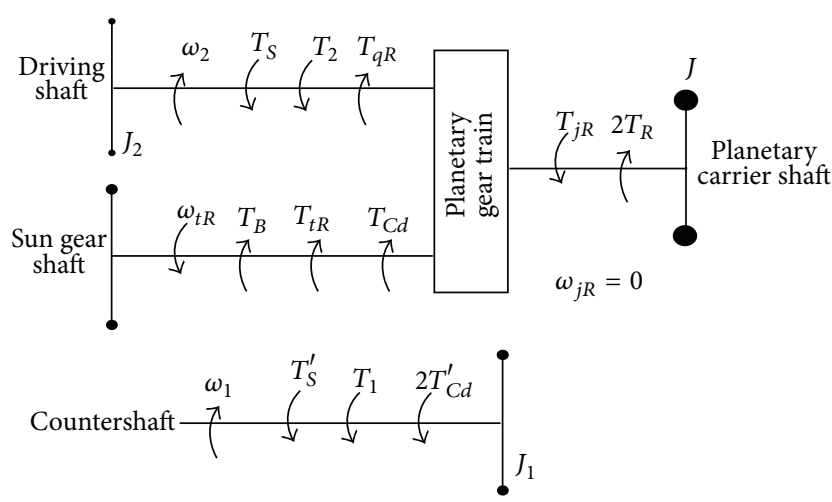

FIGURE 9: The diagram of the equivalent kinematics and dynamics analysis of the components of the gear box.

engaged, the synchronization torque and the slipping work can be reduced by extending the time before synchronization, but it will cause a longer shifting time.

2.2.2. Analysis of Shifting Process When Steering Brakes in Condition of Combination. When the steering brakes of both sides are engaged, the transitive relation between the counter shaft, and the sun gear is cut off. The motion state and stress state of each transmission component can be seen in Figure 8.

Establishing the kinetics equations of the counter shaft, the sun gear shaft and the driving shaft according to the force analysis of Figures 8 and 9, the angular speeds $\left(\dot{\omega}_{1 t 2}, \dot{\omega}_{2 t 2}\right)$ of the counter shaft, and the driving shaft in this stage are calculated:

$$
\begin{aligned}
& {\left[\begin{array}{l}
\dot{\omega}_{1 t 2} \\
\dot{\omega}_{2 t 2}
\end{array}\right]} \\
& =-\left[\begin{array}{ccccc}
\frac{1}{J_{1}} & 0 & 0 & \frac{2}{i_{z} J_{1}} & \frac{1}{i_{2} J_{1}} \\
0 & \frac{1}{\left(J_{2}+k^{2} J_{t}\right)} & \frac{k}{\left(J_{2}+k^{2} J_{t}\right)} & \frac{2 k}{\left(J_{2}+k^{2} J_{t}\right)} & \frac{1}{\left(J_{2}+k^{2} J_{t}\right)}
\end{array}\right] \\
& \times\left[\begin{array}{c}
T_{1} \\
T_{2} \\
T_{B} \\
T_{C d} \\
T_{s}
\end{array}\right]
\end{aligned}
$$

When the steering brakes are engaged, the synchronization time $\left(t_{01}, t_{12}\right)$ of the beginning and ending stage can be calculated in a similar way. And we can also obtain the functional relationship of the synchronizing torque, the angular speed of the counter shaft and the driving shaft when the synchronization begins:

$$
\begin{aligned}
& t_{01}=\left\{\begin{array}{l}
\frac{\left(\omega_{1 t 0}-\omega_{1 t 1}\right)}{\left(T_{1} / J_{1}+2 T_{C d} / i_{z} J_{1}\right)} \\
\frac{\left(\omega_{2 t 0}-\omega_{2 t 1}\right)\left(J_{2}+k^{2} J_{t}\right)}{\left(T_{2}+k T_{B}+2 k T_{C d}\right)},
\end{array}\right. \\
& t_{12}=\left\{\begin{array}{l}
\frac{\omega_{1 t 1}}{\left[T_{1} / J_{1}+2 T_{C d} /\left(i_{z} J_{1}\right)+T_{s} /\left(i_{2} J_{1}\right)\right]}, \\
\frac{\omega_{2 t 1}\left(J_{2}+k^{2} J_{t}\right)}{\left(T_{2}+k T_{B}+2 k T_{C d}+T_{s}\right)},
\end{array}\right. \\
& T_{s}=\left\{\begin{array}{l}
\frac{i_{2} J_{1} \omega_{1 t 1}}{t_{12}}-\left[i_{2} \frac{2 i_{2}}{i_{z}}\right]\left[\begin{array}{c}
T_{1} \\
T_{C d}
\end{array}\right], \\
\frac{\omega_{2 t 1}\left(J_{2}+k^{2} J_{t}\right)}{t_{12}}-\left[\begin{array}{ll}
1 & k
\end{array}\right]\left[\begin{array}{c}
T_{2} \\
T_{B} \\
T_{C d}
\end{array}\right] .
\end{array}\right.
\end{aligned}
$$

Based on energy conservation law, the fraction work produced in the process of synchronization can be calculated:

$$
\begin{aligned}
W_{s}= & \frac{1}{2} J_{1} \omega_{1 t 1}^{2}+\frac{1}{2} J_{2} \omega_{2 t 1}^{2}-\left[T_{1} \omega_{1 t 1}+\left(T_{2}+T_{B}\right) \omega_{2 t 1}\right] \\
& -\int_{t 1}^{t 2}\left(\frac{2 T_{C d} \omega_{1 t 1}}{i_{z}}\right) d t .
\end{aligned}
$$

When the steering brakes are engaged and the steering clutches are in combination condition, the influence on synchronizing torque and slipping work at the beginning of synchronization is the same, which is affected by the counter shaft and driving shaft's angular speeds and the synchronization time.

2.2.3. Comparison and Analysis of the Two Types of Shifting Processes. By comparing (12) with (17), it is obvious that when the angular speed of the counter shaft is the same as the angular speed $\left(\omega_{1 t 1}\right)$ of synchronization starting, the steering brakes in the engagement condition takes less time than the steering clutches in the engagement condition. Since $T_{B} \gg$ $T_{1}>T_{2}$, the angular speed of the driving shaft when the steering brakes are engaged is less than that in $t_{1}$, which is equivalent to reducing the speed difference of the initiative and passive parts of the synchronizer.

Comparing (14) with (19), the conclusion that the synchronizing torque in the steering brakes engaged condition is less than that in the steering clutches engaged condition when the synchronous time and the angular speed of the counter shaft are the same can be obtained.

Comparing (15) with (20), it is noticeable that the fraction work in the steering brakes engaged condition is less than that in the steering clutches engaged condition when the initial angular speeds of the counter shaft are the same $\left(\omega_{1 t 1}\right)$ in both conditions. 


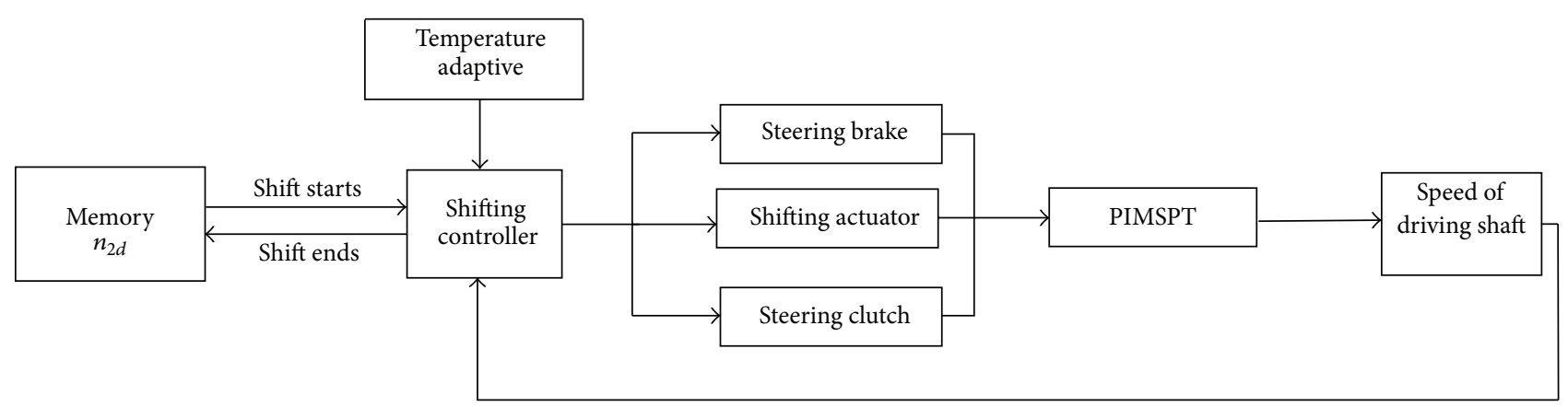

FIGURE 10: Diagram of control block of shifting into 1st gear.

To sum up, during the shifting process of starting gear, the shift time, the shift force, and the fraction work of the synchronizer can be reduced by breaking the steering brakes of both sides. As a result, the shift quality can be improved.

\section{Shifting Control Method for Staring Gear}

Aiming at optimizing the shifting control of an automatic mechanical transmission (AMT) used in vehicles, Yang et al. proposed a method of optimal shifting control based on pattern recognition and a learning algorithm [4]. Bóka et al. use a simple mechanical model to define the reduction of the speed difference between the synchronizers in order to obtain a smooth gear shifting [5]. Qi et al. analyze the principle of gear shifting in a hydraulic system and obtain an expert PID control to reduce the shifting vibration and time [6].

But the above researches and other relative researches [711] are only validated through simulation and limited to the traditional manual transmission which is not satisfied with the PIMSPT.

According to the analysis of Section 2, the requirements for steering clutch and brake control might be different depending on the different starting gears. So the shifting control strategies of two starting gears are introduced, respectively.

\subsection{Shifting Control Strategy of First Gear}

3.1.1. Selection of the Control Parameters. By controlling the steering brakes and imposing braking torque to the sun gear, the absolute value of the driving shaft angular velocity can be increased and the time of the driving shaft achieving ideal shifting rotation speed can be shortened. However, if the driving shaft rotation speed is too low during shifting, it may cause the sliding teeth sleeve to stop because the teeth of synchronization loop makes shifting difficult. Therefore, the key of the control process is to choose an appropriate shifting rotation speed of the driving shaft.

The driving shaft rotation speed and rotational acceleration can be used to describe the status and trend of driving shaft quickly and accurately, and they can be obtained by finite difference method. So, the driving shaft rotation speed and its derivative are used as the control parameters in the control process.
3.1.2. The Stage of Steering Brake Control. By imposing braking torque to the sun gear, the driving shaft rotation speed will slow down to an expected value with the control of the steering brakes. The value is inverse proportional to the temperature of transmission oil, and the relationship function can be calibrated by test. Due to the large reserve coefficient of brake, improper control might make the driving shaft rotation speed slow down to zero quickly. To address the issue and get a good performance, the incremental PD control can be adopted in the deceleration process. In the future, some advanced control methods such as robust control [12-14] can be applied.

3.1.3. The Stage of Shifting Control. This phase needs to control steering brakes and remove the braking torque imposed on the sun gear and meanwhile maintain the steering clutch separated. Steering clutch can be controlled to separate completely; at this time the sun gear of confluence planetary row is free. The planet carrier connects with the vehicle, and the degree of freedom of planetary row can be considered as one. When the sliding gear sleeve engages into the tooth, the driving shaft speed $n_{2}$ changes directly in response to the disturbance of the sun gear and it will not impact the vehicle. Therefore, steering clutch displacement is selected as a shift operation trigger in this stage.

The whole control process diagram is shown in Figure 10. The control process flow diagram is shown in Figure 11, $l s t_{\text {disengage }}$ is the steering clutch displacement, $t x$ is the shifting piston displacement, and $t x_{\min }$ is the piston displacement after shifting to 1st gear.

3.2. Shifting Control Strategy of Second Gear. When the gear shift is in the neutral position, the power flows as follows: by twice outer meshing between splitter gear and planetary gear of confluence planet row, the counter shaft transmits the torque to the driving shaft, making the driving shaft and the counter shaft rotate in the same direction, as shown in Figure 12A. However, after shifting to 2 nd gear, the rotation of driving shaft and counter shaft is in the opposite direction, as shown in Figure 12C. The direction of rotation of each axis is as follows: rotation direction of counter shaft $\omega_{1}$ and the sun gear planetary row $\omega_{t}$ must be the same, but they are the outer meshing and the direction of rotation is different; the steering clutch should be ensured that they are separated. 


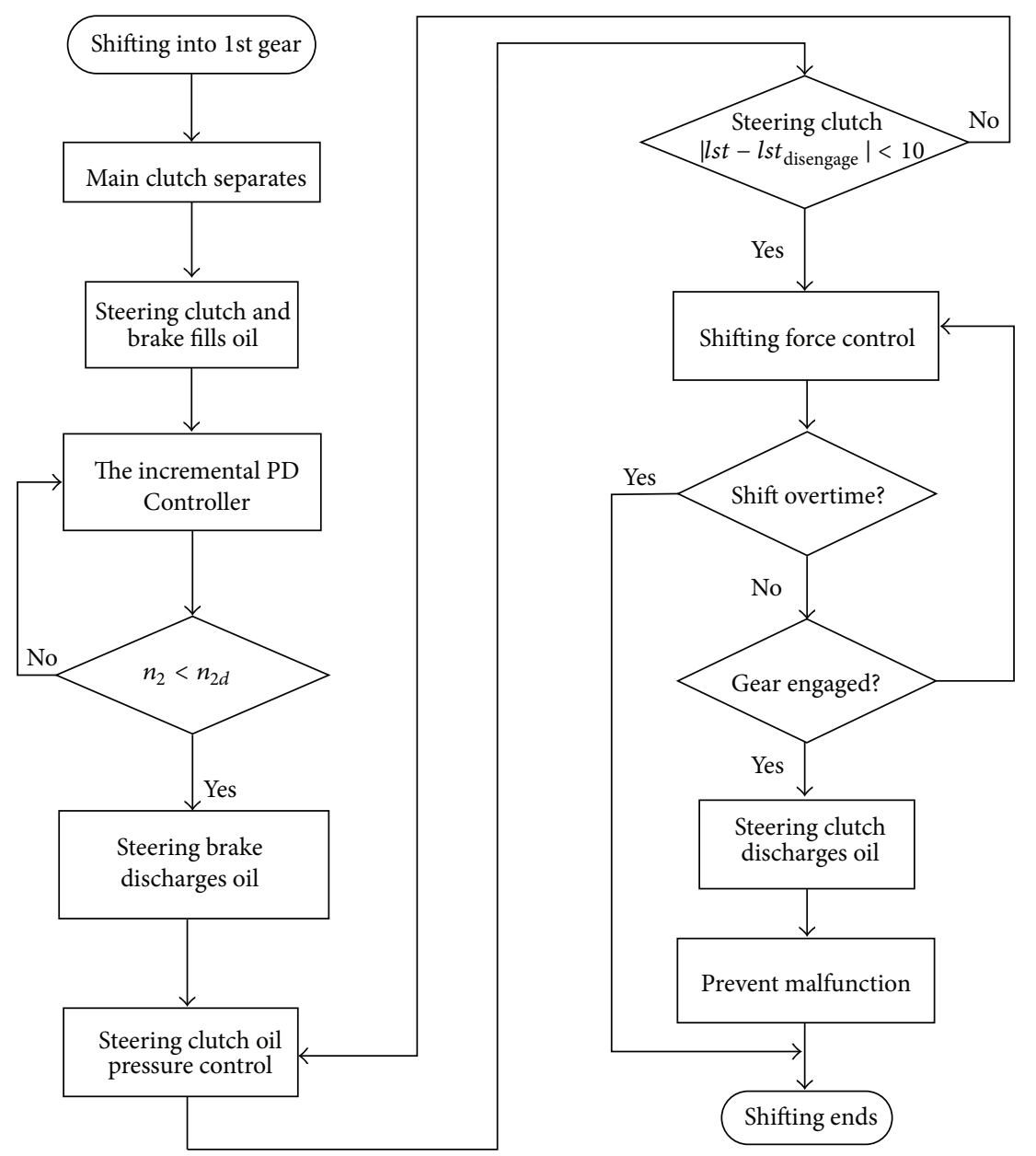

FIGURE 11: Diagram of control flow of shifting into 1st gear.
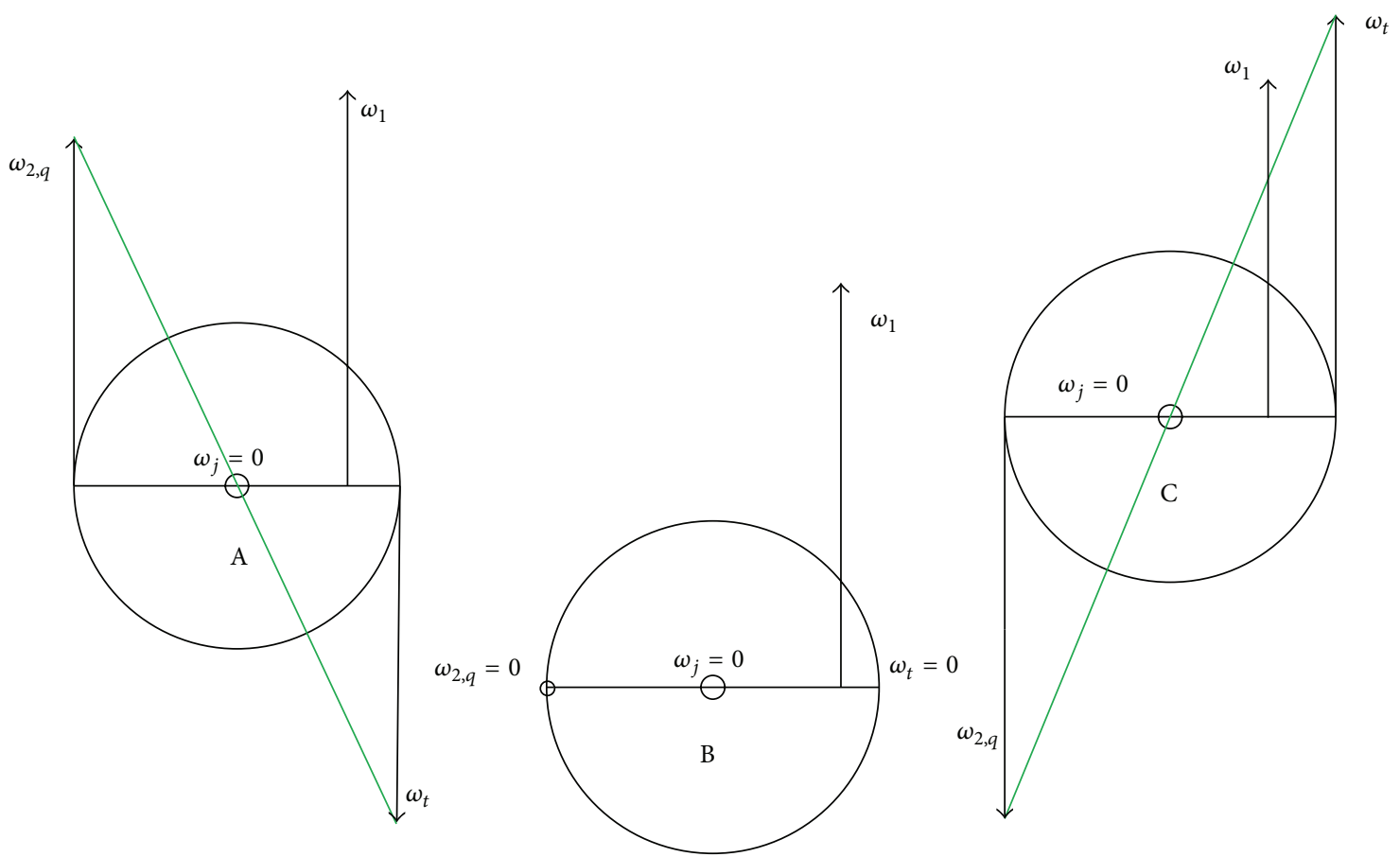

FIGURE 12: Three components velocity direction of planetary line schematic. 


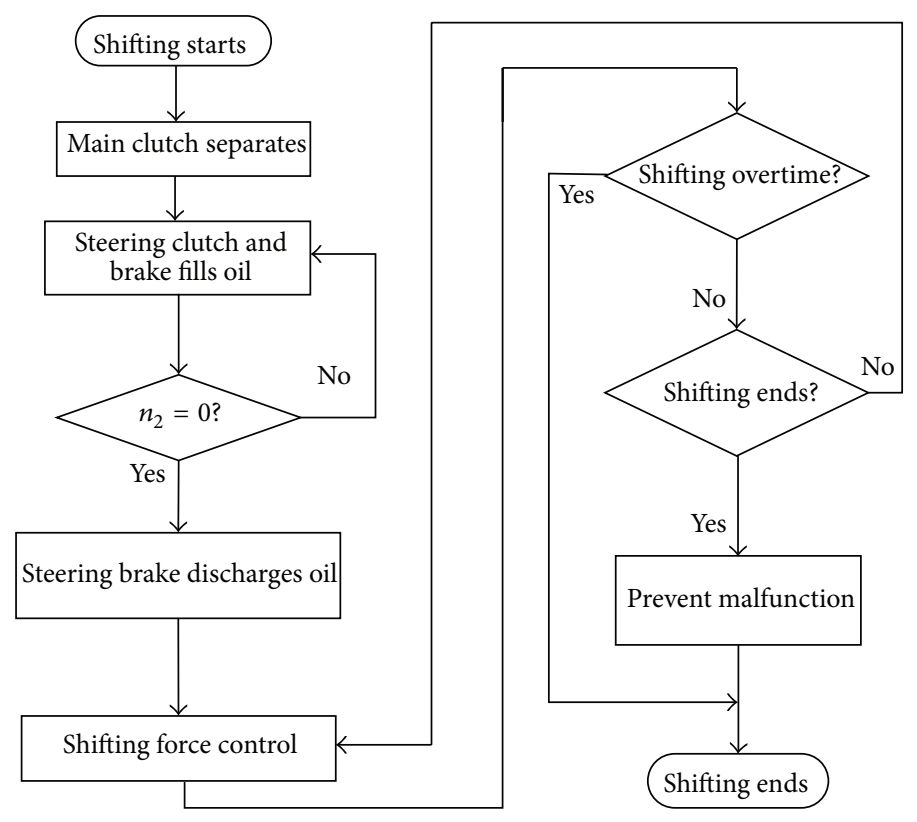

FIGURE 13: Diagram of control flow of shifting into 2nd gear.

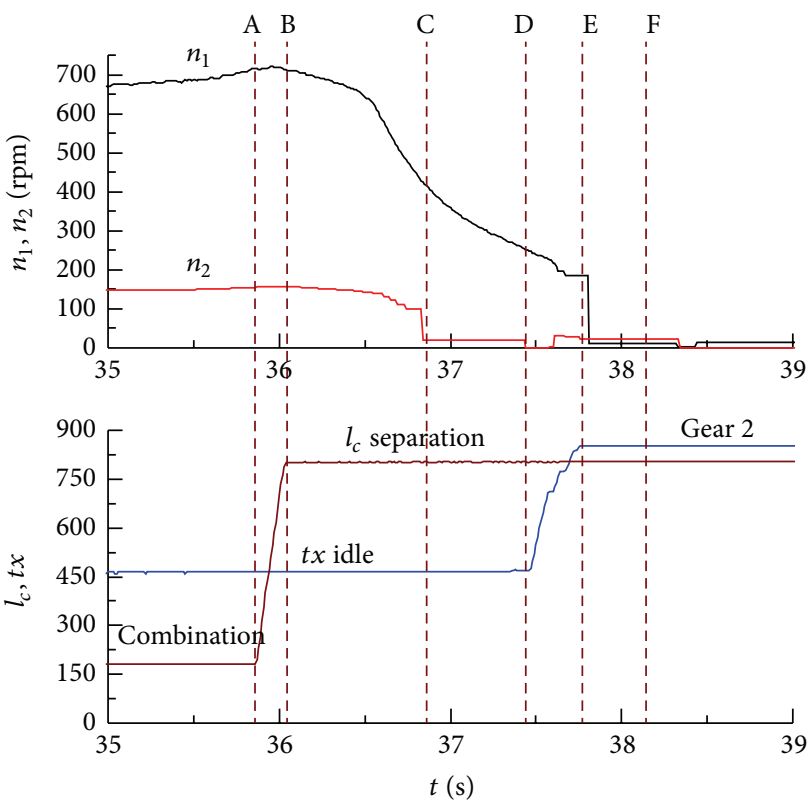

FIGURE 14: The shifting process with the controlling method.

When shifting to 2 nd gear, the rotation direction of driving shaft (ring gear wheel) $\omega_{2, q}$ changes from A to C, and it must pass through the state $\mathrm{B}$, as shown in Figure 12. In order to reduce the shifting time, steering clutch can be separated before shifting, and the maximum braking torque is imposed to the sun gear by controlling steering brake to make sure that the driving shaft speed is reduced to zero quickly, as shown in Figure 12B.

Then, a shifting action can be made after steering clutch separating completely. The diagram of control flow of shifting starting 2nd gear is shown in Figure 13.

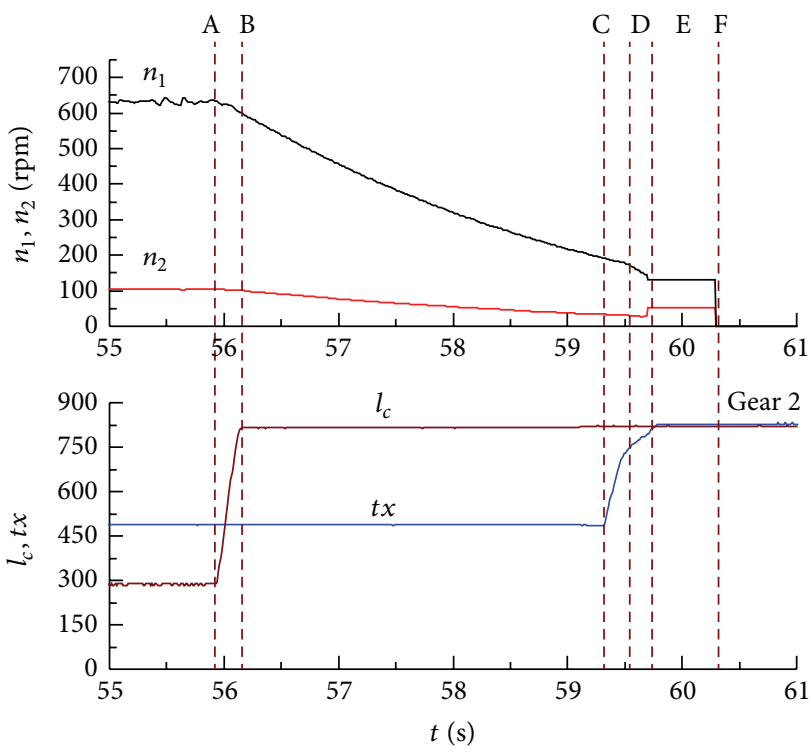

FIGURE 15: The shifting process without the controlling strategy.

\section{Experimental Researches}

The automatic shift control system for the PIMSPT was designed $[15,16]$. Experiments of shifting process of 2 nd gear are conducted with and without the strategy of controlling the steering clutches and steering brakes. The curves of the process can be seen in Figures 14 and 15.

In Figure 14, $\mathrm{A} \sim \mathrm{B}$ represents the separating process of the main clutch; $B \sim D$ represents the decline stage of the angular speed of driving shaft; $B \sim C$ represents the response time of the control stage; $\mathrm{D} \sim \mathrm{E}$ represents the oil charging stage of 
hydrocylinder during shifting process and the stage of eliminating synchronizer gap; E F represents the accomplishment of shifting action with synchronizer working.

In Figure 15, $\mathrm{A} \sim \mathrm{B}$ represents the separating process of the main clutch; $\mathrm{B} \sim \mathrm{C}$ represents the decline stage of the angular speeds of driving and counter shaft to a reasonable shift speed; $\mathrm{C} \sim \mathrm{E}$ represents the oil charging stage of hydrocylinder during shift process and the stage of eliminating synchronizer gap; E F represents the accomplishment of shifting action with synchronizer working.

Comparing Figure 14 with Figure 15, it is obvious that the stage E F of Figure 14 parallels the stage D E of Figure 15. From the figures, we can make a conclusion that when the shifting jerks are the same, the shifting time could be reduced over $50 \%$ by adopting the new shifting control strategy.

\section{Conclusions}

As the main clutch of the positive independent mechanical split path transmission is released, the speed of countershaft and driving shaft can be descended faster by controlling the steering clutches and brakes. Based on this mechanism, the shifting time can be shortened and the shifting jerk can be reduced as well with steering clutches and brakes control, which take the driving shaft speed as a closed-loop control parameter and can actively control the speed difference between the driving part and passive part of synchronizer. Subsequently, this control strategy has been approved with a road test of vehicle that it can improve the shift quality effectively.

\section{References}

[1] J.-M. Meng, J.-Q. Xi, H.-Y. Chen, and P. Gong, "Automatic shift technology for positive independent mechanical split path transmission," Acta Armamentarii, vol. 29, no. 1, pp. 2-5, 2008.

[2] J.-M. Meng, J.-Q. Xi, and H.-Y. Chen, "Automated shift mechanism applied to AMT system of positive independent mechanical split path transmission," Transactions of Beijing Institute of Technology, vol. 2, no. 2, pp. 108-112, 2009 (Chinese).

[3] Y.-Y. Chen, J.-Q. Xi, and H.-Y. Chen, "Optimal gear shift control strategy for positive independent mechanical split path transmission with automatic shift system," Journal of Beijing Institute of Technology, vol. 20, no. 3, pp. 387-393, 2011.

[4] Z. Yang, C. Cao, and Y. Su, "A method of optimal shift control based on pattern recognition and learning algorithm," in Proceedings of the 4th World Congress on Intelligent Control and Automation, vol. 2, pp. 955-959, Shanghai ,China, June 2002.

[5] G. Bóka, J. Márialigeti, L. Lovas, and B. Trencséni, "Face dog clutch engagement at low mismatch speed," Periodica Polytechnica Transportation Engineering, vol. 38, no. 1, pp. 2935, 2010.

[6] S. Qi, C. Zhang, and H. Xie, "SOPC technology applied on automatic mechanical transmission control unit design," in Proceedings of the IEEE/ASME International Conference on Mechatronic and Embedded Systems and Applications, pp. 529532, Qingdao, China, 2010.

[7] Z. Zhong, G. Kong, Z. Yu, X. Xin, and X. Chen, "Shifting control of an automated mechanical transmission without using the clutch," International Journal of Automotive Technology, vol. 13, no. 3, pp. 487-496, 2012.

[8] C. H. Yu and C. Y. Tseng, "Research on gear-change control technology for the clutchless automatic-manual transmission of an electric vehicle," Journal of Automobile Engineering, vol. 223, no. 9, pp. 1101-1108, 2013.

[9] H. Liu, Y. Lei, Z. Li, J. Zhang, and Y. Li, "Gear-shift strategy for a clutchless automated manual transmission in battery electric vehicles," SAE International Journal of Commercial Vehicles, vol. 5, no. 1, pp. 57-62, 2012.

[10] G. Lucente, M. Montanari, and C. Rossi, "Modelling of an automated manual transmission system," Mechatronics, vol. 17, no. 2-3, pp. 73-91, 2007.

[11] L. Glielmo, L. Iannelli, V. Vacca, and F. Vasca, "Gearshift control for automated manual transmissions," Mechatronics, vol. 11, no. 1, pp. 17-26, 2006.

[12] H. Zhang, Y. Shi, and J. Wang, "Observer-based tracking controller design for networked predictive control systems with uncertain Markov delays," International Journal of Control, vol. 86, no. 10, pp. 1824-1836, 2013.

[13] H. Zhang, Y. Shi, and M. Liu, "H ${ }_{\infty}$ step tracking control for networked discrete-time nonlinear systems with integral and predictive actions," IEEE Transactions on Industrial Informatics, vol. 9, no. 1, pp. 337-345, 2013.

[14] H. Zhang, Y. Shi, and A. Saadat Mehr, "Robust static output feedback control and remote PID design for networked motor systems," IEEE Transactions on Industrial Electronics, vol. 58, no. 12, pp. 5396-5405, 2011.

[15] J. Xi, H. Chen, and J. Meng, "Automatic mechanical transmission used for tracked vehicle equipped with fixed shaft transmission," Chinese Patent, China, CN101539200, 2009.

[16] J. Xi, J. Meng, and Y. Zhai, "The automatic shifting control for positive independent mechanical split path transmission system," Chinese Patent, China, CN101695903A, 2010. 


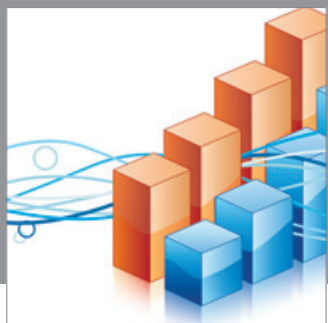

Advances in

Operations Research

mansans

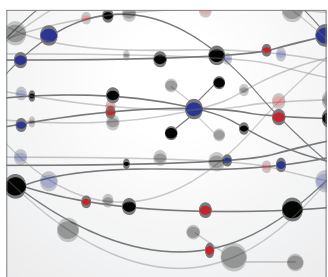

The Scientific World Journal
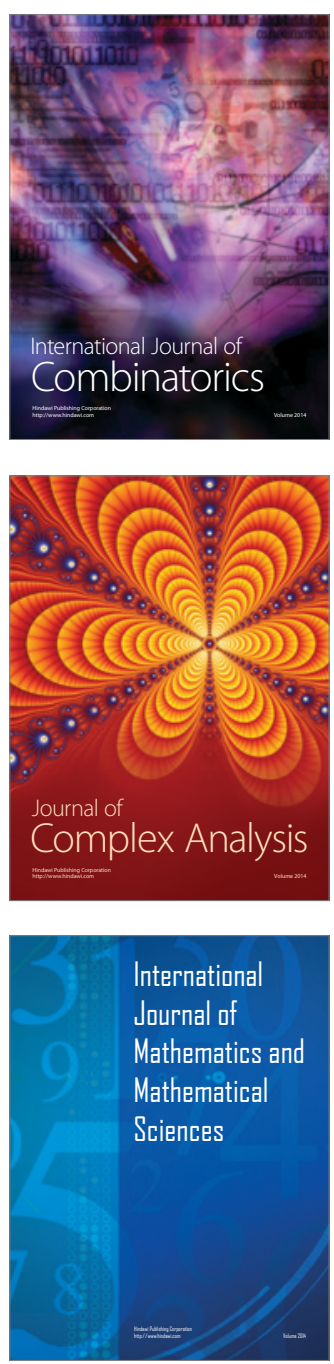
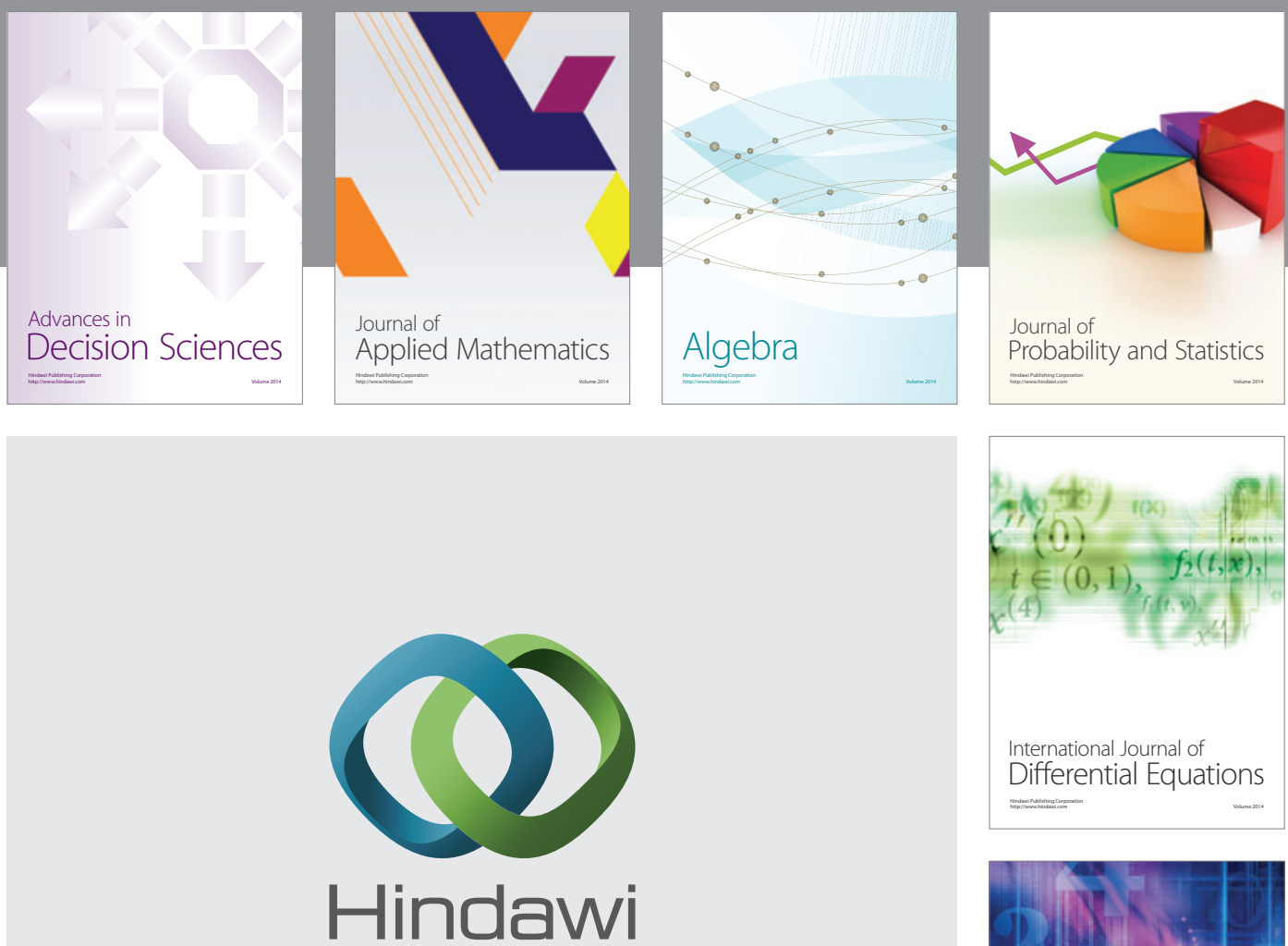

Submit your manuscripts at http://www.hindawi.com
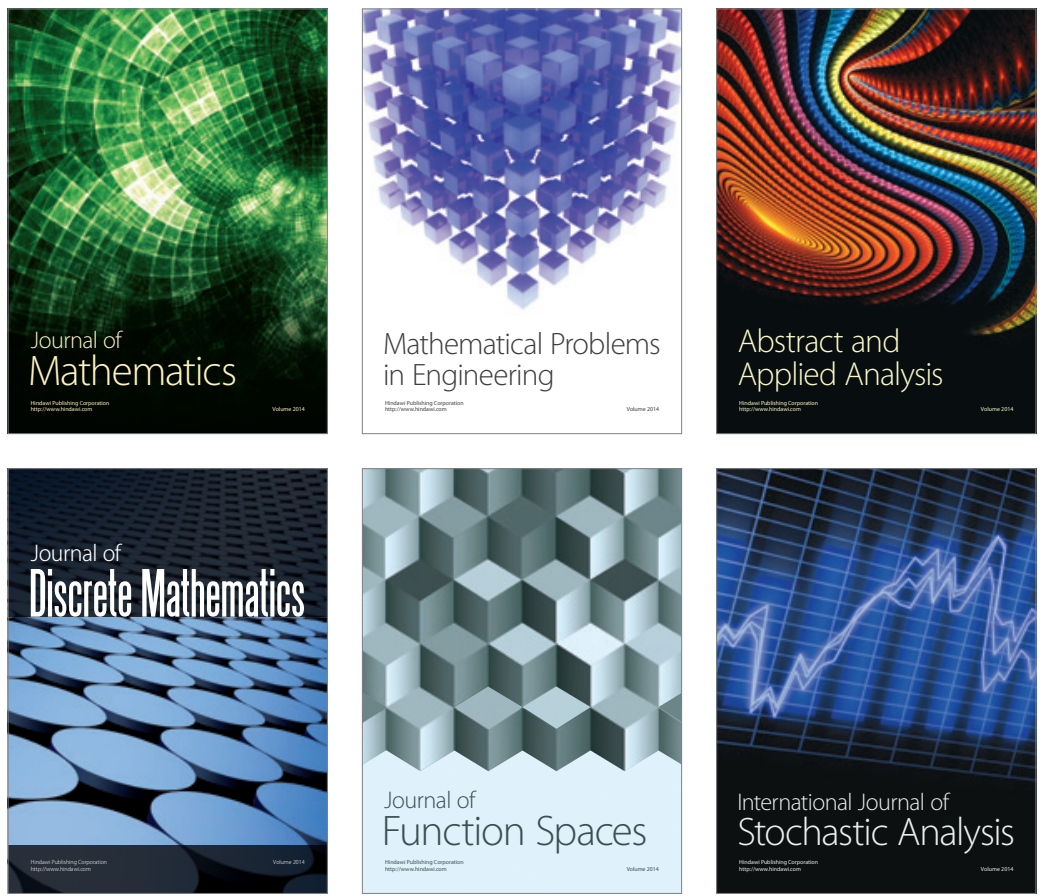

Journal of

Function Spaces

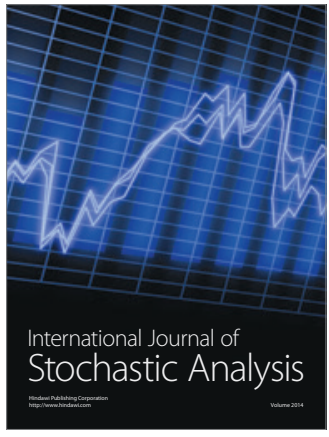

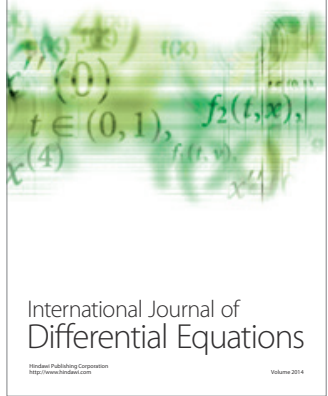
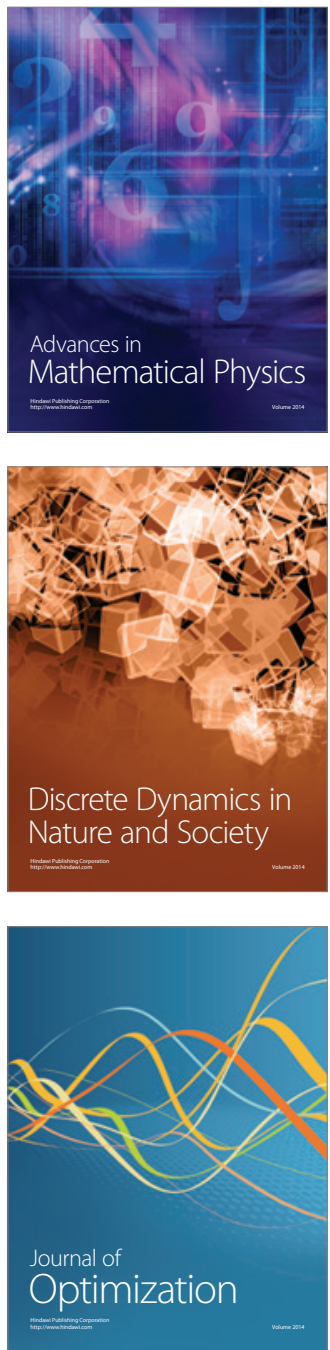